\title{
Analysing the genetic architecture of clubroot resistance variation in Brassica napus by associative transcriptomics
}

\author{
Ondrej Hejna • Lenka Havlickova • Zhesi He • \\ Ian Bancroft $\mathbb{D} \cdot$ Vladislav Curn
}

Received: 6 February 2019 / Accepted: 8 July 2019/Published online: 20 July 2019

(C) The Author(s) 2019

\begin{abstract}
Clubroot is a destructive soil-borne pathogen of Brassicaceae that causes significant recurrent reductions in yield of cruciferous crops. Although there is some resistance in oilseed rape (a crop type of the species Brassica napus), the genetic basis of that resistance is poorly understood. In this study, we used an associative transcriptomics approach to elucidate the genetic basis of resistance to clubroot pathotype ECD $17 / 31 / 31$ across a genetic diversity panel of 245 accessions of $B$. napus. A single nucleotide polymorphism (SNP) association analysis was performed with 256,397 SNPs distributed across the genome of B. napus and combined with transcript abundance data of 53,889 coding DNA sequence (CDS) gene models. The SNP association analysis identified two major loci (on chromosomes A2 and A3) controlling resistance and seven minor loci. Within these were a total of 86 SNP markers. Altogether, 392 genes were found in these regions. Another 21 genes were implicated as potentially involved in resistance using gene expression marker
\end{abstract}

Electronic supplementary material The online version of this article (https://doi.org/10.1007/s11032-019-1021-4) contains supplementary material, which is available to authorized users.

O. Hejna $\cdot$ V. Curn

Biotechnological Centre, Faculty of Agriculture, University of South Bohemia, Studentska, 1668 Ceske Budejovice,

Czech Republic

O. Hejna · L. Havlickova · Z. He · I. Bancroft $(\bowtie)$ Department of Biology, University of York, Heslington, York YO10 5DD, UK

e-mail: ian.bancroft@york.ac.uk
(GEM) analysis. After GO enrichment analysis and InterPro functional analysis of the identified genes, 82 candidate genes were identified as having roles in clubroot resistance. These results provide useful information for marker-assisted breeding which could lead to acceleration of pyramiding of multiple clubroot resistance genes in new varieties.

Keywords Association genetics - Brassica napus . Clubroot · Transcriptomics

\section{Introduction}

Clubroot disease caused by the soil-borne obligate biotrophic pathogen Plasmodiophora brassicae is one of the most serious diseases of cruciferous crops, especially the allotetraploid Brassica napus $(2 n=38$, AACC) and its diploid progenitor species Brassica rapa $(2 n=$ 20, AA) and Brassica oleracea $(2 n=18, \mathrm{CC})$ (Dixon 2009; Fredua-Agyeman and Rahman 2016). Two-phase infection by this parasite leads to formation of galls or clubs on the roots and hypocotyls of diseased plants (Hwang et al. 2012). Creation of these club-shaped roots ultimately leads to the interruption of the uptake and flow of water and minerals in roots. This results in wilting, stunted growth, chlorosis and leaf abscission leading even to the death of infected plants (Kageyama and Asano 2009). The symptoms of clubroot disease result in significant reduction of seed yield as well as decreasing of oil content in seed of susceptible cruciferous plants worldwide (Dixon 2009). The average loss of yield in 
areas with $P$. brassicae exceeds $20 \%$ but may lead to total crop failure (Pageau et al. 2006). Indeed, the evergrowing rapeseed production all over the world in the past years also increase areas infested by $P$. brassicae. Dissemination has been observed in Europe, China, India, Canada and Australia (Diederichsen et al. 2014; Chai et al. 2014; Bhattacharya et al. 2014; Rahman et al. 2014; Donald and Porter 2014).

Three main systems have been set to distinguish between pathotypes of $P$. brassicae. The most commonly used is the Williams classification (Williams 1966). This system is based on 4 different resistant varieties and is able to distinguish 16 different pathotypes. Seven different pathotypes have been revealed in the Czech Republic. The most common pathotypes are 7 (43\%) and 6 (29\%) (Ricarova et al. 2016). Other systems distinguishing pathotypes are the European clubroot differential (ECD) set (Buczacki et al. 1975) and the differential system of Some et al. (1996).

Traditional agricultural practices such as soil liming or agrochemical treatments by fungicides are expensive and not very effective to control this disease (Hwang et al. 2014), which is further complicated due to the broad range of hosts in which the pathogen is able to reproduce and the ability of spores to survive more than 20 years (Wallenhammar 1996). The most effective and economic strategy to eliminate clubroot disease is a combination of conventional disease-management measures including crop rotation, liming, application of fungicides and disinfection of equipment in combination with the use of varieties possessing multi-resistant genes (Faggian and Strelkov 2009).

Intensive breeding of cultivars resistant to clubroot has been ongoing for many years. The main sources of clubroot resistance (CR) genes were found in European fodder turnip cultivars (Brassica rapa), namely Gelria R, Siloga, Debra and Milan White (Hirai 2006). These sources of resistance were successfully introgressed into Brassica napus, which has led to the release of several resistant rapeseed cultivars (Piao et al. 2009).

The effort of scientists to find resistance genes is increasing every year along with the growing areas affected by $P$. brassicae. Recently, two CR genes in B. rapa have been cloned: $C R a$ has been mapped on chromosome A3 (Ueno et al. 2012) and Crrl on chromosome A8 (Hatakeyama et al. 2013). Furthermore, seven other loci have been finely mapped on the A3 chromosome of B. rapa, namely CRb, CRb-kato, CRd, Crr3, Rcr1, Rcr2 and Rcr4 (Saito et al. 2006; Kato et al.
2013; Chu et al. 2014; Zhang et al. 2014; Huang et al. 2017; Yu et al. 2017; Pang et al. 2018). At least ten another CR loci were discovered in the A genome; $\mathrm{Cr} 2$ was mapped on A1 (Suwabe et al. 2003); $C R c$ and $R c r 8$ on A2 (Sakamoto et al. 2008; Yu et al. 2017); $C R k$, PbBa3.1 and PbBa3.3 on A3 (Sakamoto et al. 2008; Chen et al. 2013); CrrA5 on A5 (Nguyen et al. 2018); Crr4 on A6 (Suwabe et al. 2006); and Rcr9 on A8 (Yu et al. 2017).

In contrast with $B$. rapa, less progress has been made towards the identification of $\mathrm{CR}$ genes in the $B$. oleracea genome (C genome). Previous studies have presumed that there are much less dominant $\mathrm{CR}$ genes in $\mathrm{C}$ genome and $\mathrm{CR}$ is quantitative under polygenic control there (Zhang et al. 2016). So far, five loci have been described: $C R 2 a, C R 2 b, P b 3, P b 4$ and PbBol (Landry et al. 1992; Grandclément and Thomas 1996; Voorrips et al. 1997; Rocherieux et al. 2004). Furthermore, at least $10 \mathrm{CR}$ quantitative trait loci (QTL) have been mapped in the $\mathrm{C}$ genome: $Q T L$ $L G 3$ on $\mathrm{C} 1$ (Nomura et al. 2005), Pb-Anju1, PbAnju2 and CRQTL-YC on C2 (Nagaoka et al. 2010; Lee et al. 2016), Pb-Anju3 on C3 (Nagaoka et al. 2010), $P b-G C 1$ and $Q T L-L G 9$ on C5 (Nagaoka et al. 2010; Nomura et al. 2005) and Pb-Anju4 and Rcr7 on C7 (Nagaoka et al. 2010; Dakouri et al. 2018).

Currently, more than $30 \mathrm{CR}$ loci and two dominant $\mathrm{CR}$ genes have been proposed in the $\mathrm{AC}$ genome of B. napus. Manzanares-Dauleux et al. (2000) discovered one dominant gene $\mathrm{Pb}-\mathrm{Bn} 1$ on A4 and two QTLs on A4 and C5. So far, the most CR loci have been mapped by Werner et al. (2008), in which 19 QTL (most racespecific) were detected across 8 chromosomes.

The first GWAS-based study using Brassica $60 \mathrm{~K}$ SNP arrays for screening a natural population of 472 $B$. napus accessions in an infected field to detect resistance genes to most predominant pathotype 4 of P. brassicae in China mapped 10 loci on A4, A10, C3, C4, C6 and C9 chromosomes (Li et al. 2016). Compared to $60 \mathrm{~K} \mathrm{SNP}$ array, current associative transcriptomics (AT) platform (Havlickova et al. 2018) offers much better SNP coverage with the added benefit of using transcript abundance data. AT that was first described by Harper et al. (2012) has been previously used to identify genes underlying control of seed glucosinolate content (Lu et al. 2014), anion homeostasis (Koprivova et al. 2014), cell wall polysaccharides (Wood et al. 2017) and leaf calcium and magnesium accumulation (Alcock et al. 2017). 
In this study, 245 diverse B. napus genotypes were inoculated under controlled conditions by the mixture of most predominant $P$. brassicae pathotype ECD 17/31/ 31 in the Czech Republic and scored for clubroot resistance. These data were used for AT with aim to elucidate associated regions associated with a source of clubroot resistance.

\section{Materials and methods}

Plant material

A panel of 245 accessions of Brassica napus used to test clubroot resistance and subsequent association analysis has been previously reported (Havlickova et al. 2018). Based on previous relatedness characterization, accessions were defined as winter oilseed rape (101), winter fodder (4), spring oilseed rape (92), swede (17), kale (2), semi-winter (5) and not assigned crop type (24). This collection is composed of lines all over the world, including varieties from Europe, Asia, North America, Australia and North Africa, with lines released from the 1950s up to today's modern winter Canola type oilseed rape (Supplemental Table 1).

\section{Pathogen isolates}

Inoculum used for testing of the resistance to clubroot was composed by the most aggressive pathotypes P. brassicae within the Czech Republic. Clubs for inoculum preparation were collected from the hardest hit areas affected by clubroot near Svetla Hora in the Moravian-Silesian Region (Ricarova et al. 2016). According to the identification method called the ECD (European Clubroot Differential set) Buczacki et al. (1975), the pathogen was identified as pathotypes 17/31/31.

Inoculum standing spores were obtained from a solid club of infected plants. Before use, the tumours were stored at $-18{ }^{\circ} \mathrm{C}$. The clubs were pureed in distilled water for $3 \mathrm{~min}$ at the highest speed to prepare an inoculum. The final suspension was filtered through a muslin cloth and then centrifuged three times for $7 \mathrm{~min}$; the resultant clusters of spores were resuspended in distilled water and adjusted with Bürker chamber to the desired concentration of $100 \mathrm{M}$ spores in $1 \mathrm{ml}$ of inoculum.
Inoculation of spores and plant cultivation

Cultivation trays with a cell size of $4 \times 4 \mathrm{~cm}$ were filled with a mixture of coarse pearlite and conventional growing medium for vegetables (Forestina, Czech Republic) with a $\mathrm{pH} 6.5$ in the ratio $1: 1$. The seeds of tested genotypes were sown in each pot on the surface of the growth substrate. On every seed was applied by micropipette $0.5 \mathrm{ml}$ of inoculum at a concentration of $100 \mathrm{M}$ spores $\mathrm{ml}^{-1}$ and covered with $1 \mathrm{~cm}$ of coarse pearlite. As the standard, extremely susceptible variety of Chinese cabbage 'Granaat' has been used. The prepared plant pots were placed with five replications in a randomized design on trays within constantly maintained about $1 \mathrm{~cm}$ high water level. Plants were grown in a growth chamber with a 16-h day ( 80 to $100 \mu \mathrm{E} \mathrm{m}^{-2} \mathrm{~s}^{-1}$ at $\left.20{ }^{\circ} \mathrm{C}\right)$ and an 8 -h night $\left(18{ }^{\circ} \mathrm{C}\right)$ photoperiod over a period of 7 weeks. Indication of infection was observed 3 weeks after sowing. The plants were fertilized weekly using a solution of Kristalon Start (AGRO CS a.s., Czech Republic) (0.5 g per 101 of water).

\section{Evaluation of infestation}

Disease severity was assessed 7 weeks after inoculation from roots using a standard $0-3$ scale, where $0=$ no visual symptoms, $1=$ clubs only on the lateral roots, $2=$ main root clubs and $3=$ deformed entire root system (Buczacki et al. 1975) (Fig. 1a) The disease index (DI) was determined as follows:

$\mathrm{DI}=\left[\left(n_{1}+2 n_{2}+3 n_{3}\right) /\left(N_{\mathrm{T}} \times 3\right)\right] \times 100$, where $n_{1}$ to $n_{3}$ were the number of plants with different disease severity of 1-3 scale and $N_{\mathrm{T}}$ represented the total number of identified plants, respectively ( $\mathrm{Li}$ et al. 2016). Very sensitive genotypes showed DI above $80 \%$, while high resistant genotypes reveal DI less than $20 \%$.

Transcriptome sequencing, SNP detection, gene expression and associative transcriptomics

Plant material was a subset of the genetically diverse AT panel of 383 rapeseed accessions described by Havlickova et al. (2018). The growth conditions for RNA extraction, transcriptome sequencing and functional genotype determination were reported previously by $\mathrm{He}$ et al. (2017). The genotypes reported in Havlickova et al. (2018) were re-used for the present study. Mapping and associative transcriptomics were performed as described by Havlickova et al. (2018). In 
a

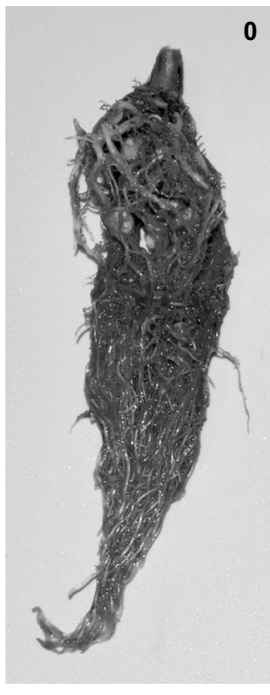

b

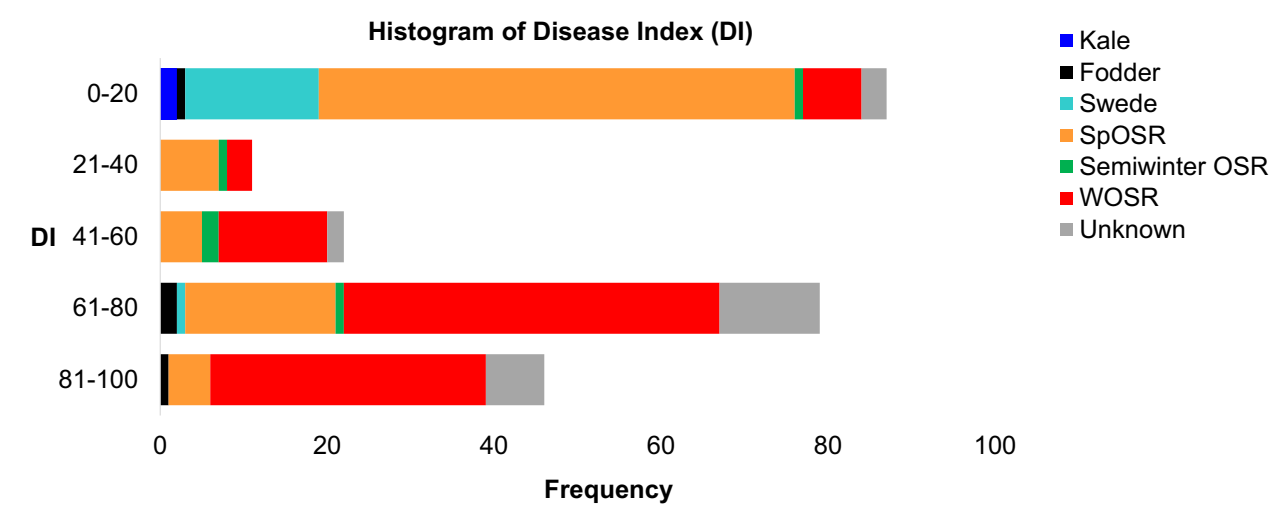

3

2

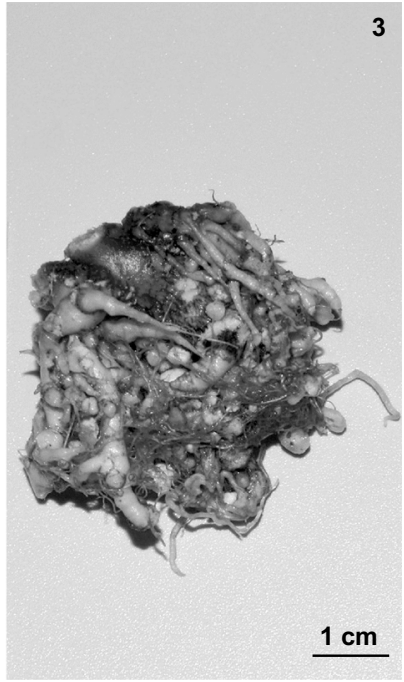

$1 \mathrm{~cm}$
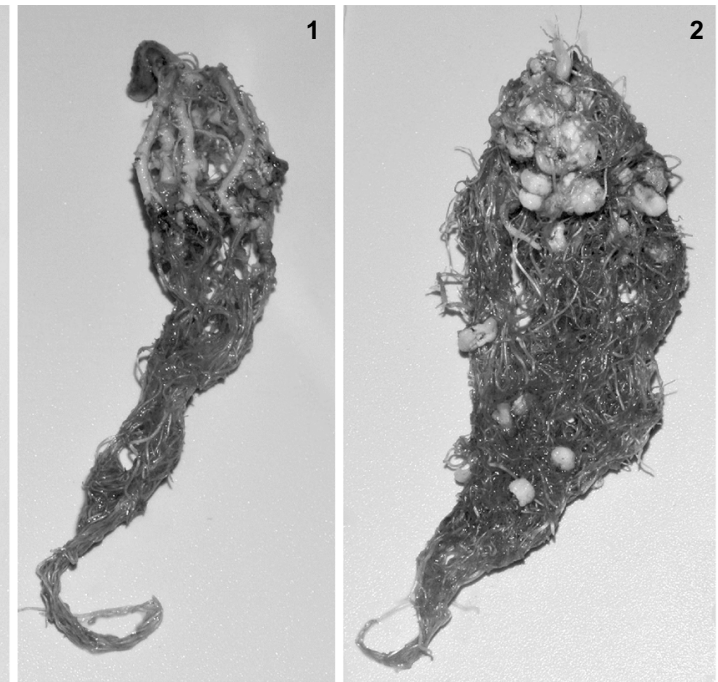

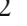


chromosome as previously described by Havlickova et al. (2018). Potential candidate genes were identified within the range of LD block regarded as region with the most significant SNPs $(-\log 10 P>4)$ which $r^{2}>0.4(\mathrm{Li}$ et al. 2016); when not present, a region of $\pm 0.2 \mathrm{Mb}$ (Samayoa et al. 2015) from the significant SNPs on the pseudomolecule reference sequence was checked for annotated genes putatively involved in plant response to the club root. To uncover potential candidate genes, GO enrichment analysis, InterPro functional analysis and manual annotation based on the similarity of A. thaliana were performed.

\section{Results}

Phenotypic variation of clubroot resistance in a diversity panel

Resistance to clubroot was assessed in an association panel of 245 Brassica napus accessions by using DI (Figs. 1b and 2; Supplemental Table 1). The measured values showed a high level of phenotypic variation within the panel. The DIs ranged from 0 to 100 with an average value of $45.51 \pm 2.32$ standard error (SE). The frequency distributions diagrams of DIs indicated a certain degree of separation between almost fully resistant lines and sensitive ones. Moreover, $35.5 \%$ of lines were classified as resistant $(\mathrm{DI}<20)$, whereas $19.6 \%$ demonstrated extreme susceptibility to the clubroot disease (DI > 80) with prevalent amount of lines with DI in the range of 61-70 (24\%). Unequal distributions of data may indicate that the resistance is driven by major resistance gene or genes accompanied by multi-loci of weaker effect. To evaluate the effect of the crop type to clubroot resistance, frequency distribution was assessed (Fig. 1b). Both kale lines were found to be resistant $(\mathrm{DI}=0)$, followed by swede $(\mathrm{DI}=3.9 \pm 3.8)$, spring OSR (DI $=23.5 \pm 3.3$ ), semiwinter OSR (DI $=43.9 \pm$ 8.8 ), fodder with largest variation $(\mathrm{DI}=56.7 \pm 17.2$ ), not assigned crop types (DI $=66.2 \pm 5.8)$ and winter OSR (DI $=68.2 \pm 2.4$ ).

SNP association analysis

AT analysis of DI identified 9 SNP association peaks with trait variation (Fig. 3). They were called according to location on the $B$. napus pseudomolecule (BnA01_0308,BnA02_0265, BnA02_0286, BnA03_0186, BnA03_0263, BnA08_0009, BnC02_0414,BnC07_0238,BnC07_0421).

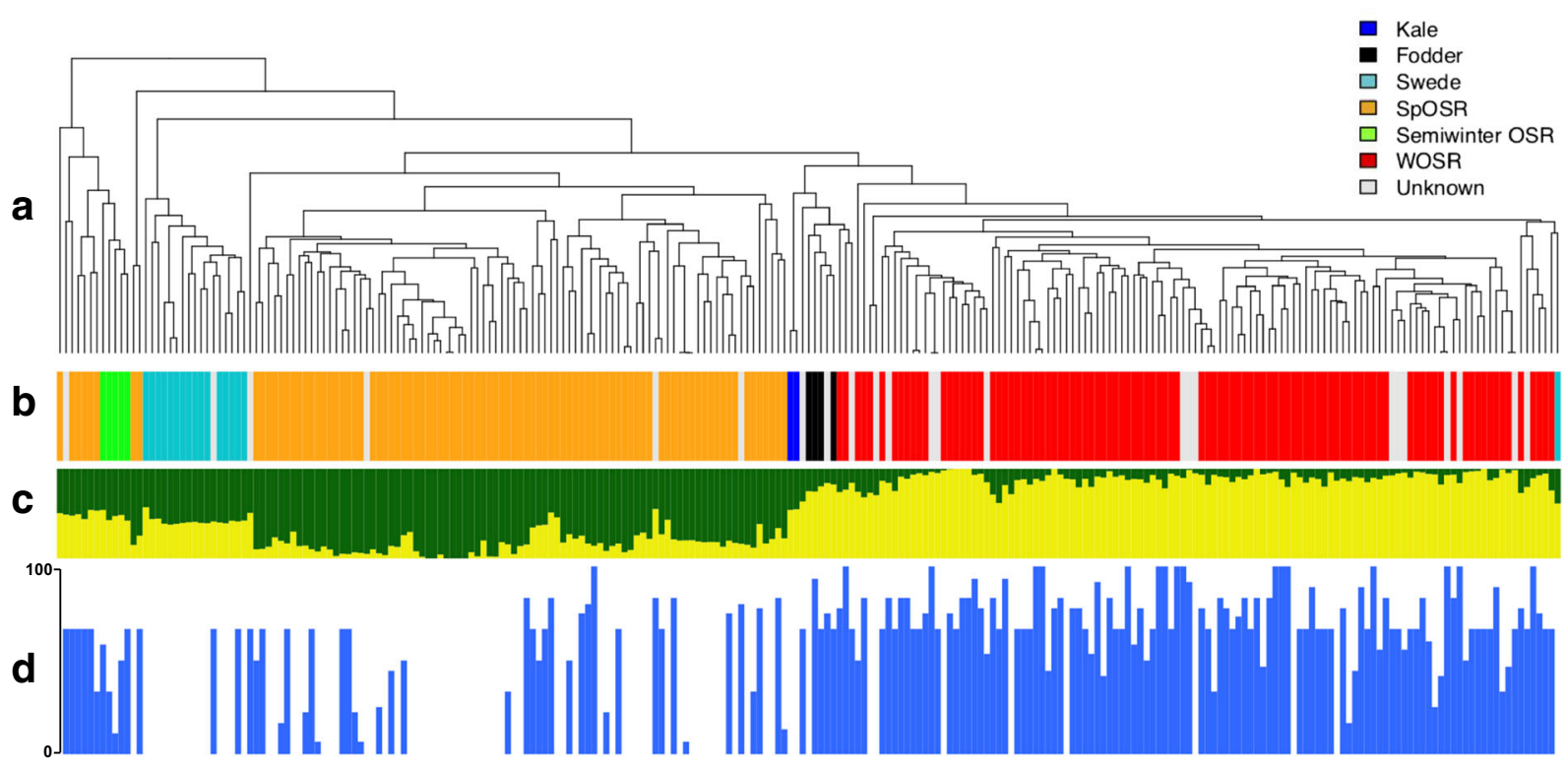

Fig. 2 Population structure and trait variation across 245 B. napus accessions. a Relatedness of accessions in the panel based on 355,536 scored SNPs. b Main crop types in the panel, colourcoded: orange for spring oilseed rape, green for semi-winter oilseed rape, light blue for swede, dark blue for kale, black for fodder and red for winter oilseed rape, grey for crop type not assigned. c Population structure for highest likelihood $K=2$. d Variation for clubroot resistance by using disease index DI $(\mathrm{DI}=0$, no visual symptoms; DI = 100, deformed entire root system) 


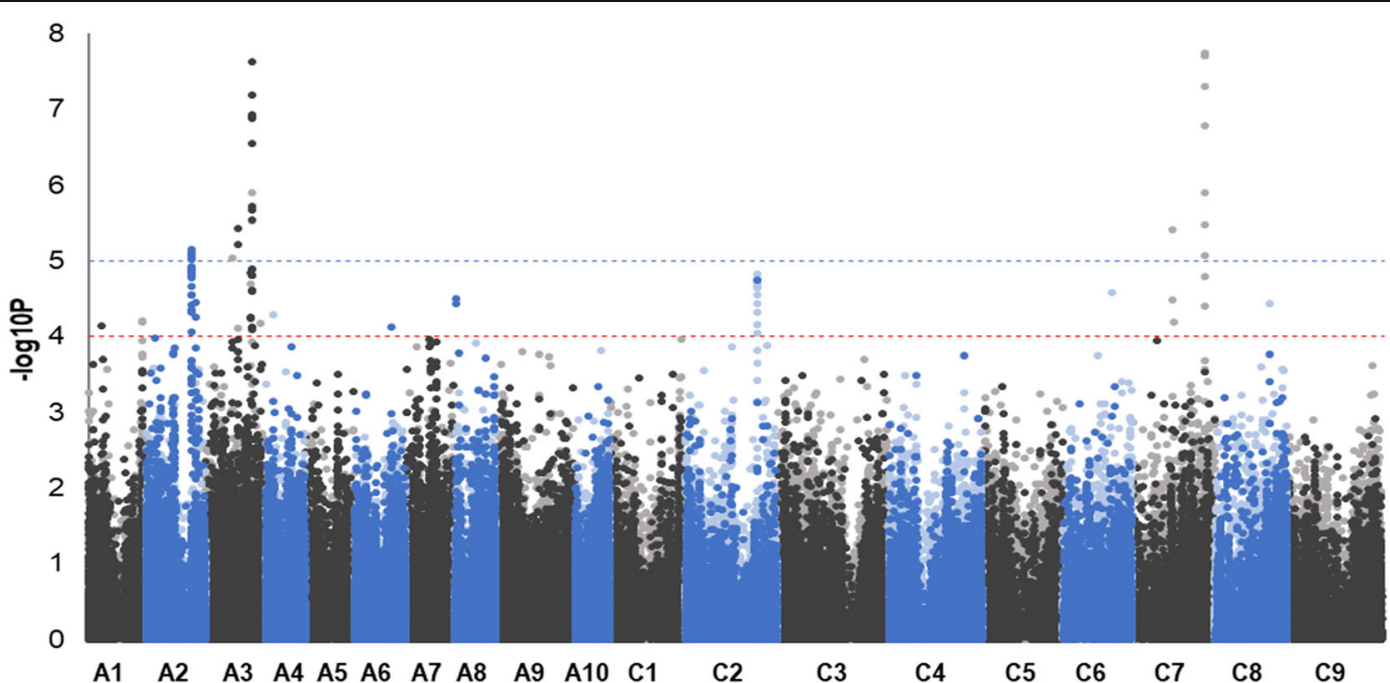

Fig. 3 Transcriptome SNP association analysis for clubroot resistance. The SNP markers are positioned on the $x$-axis based in the genomic order of the gene models in which the polymorphism was scored, with the significance of the trait association, as $-\log 10 P$, on the $y$-axis. A1 to $\mathrm{A} 10$ and $\mathrm{C} 1$ to $\mathrm{C} 9$ are the chromosomes of $B$. napus, shown in alternating black and blue colours to permit boundaries to be distinguished. Hemi-SNP markers (i.e. polymorphisms involving multiple bases called at the SNP position in one

Five SNP association peaks included sSNPs as shown in Supplemental Table 2. In total, 86 SNPs were highly associated with clubroot resistance, 29 of them were called as sSNP and the remaining 57 as pSNP. Five SNPs were lying alone above $-\log 10 P<10^{-4}$ threshold line without any close sSNPs or pSNPs to create a distinguishable peak, therefore excluded from further investigation. The rest of the associated SNPs were clustered in small loci and formed clear peaks. Graphic representation of SNP association analysis in Manhattan plots for individual chromosomes is seen in Supplemental Figure 3. The most significantly associated peak BnA03_0263 with predominant simple sSNPs ($\log 10 P>7)$ that can be assigned with confidence to a genome was discovered on chromosome A03 (Fig. 4). This peak was accompanied by the presence of corresponding shadow peak in homeologous region of chromosome C07 (BnC07_0421) shown in Supplemental Figure $3 \mathrm{~h}$. Furthermore, other associated peaks in very small regions with simple sSNPs BnA02_0265 and BnA03_0186 were found on chromosomes A02 and A03 respectively (Supplemental Figure 3b, d). Both of them were also accompanied by the presence of corresponding shadow peaks with hemi-SNPs in homeologous regions of chromosomes $\mathrm{C} 02$ and $\mathrm{C} 07$ : $B n C 02$ 0414 (Supplemental Figure 3f) and allele of the polymorphism) for which the genome of the polymorphism cannot be assigned are shown as light points, whereas simple SNP markers (i.e. polymorphisms between resolved bases) and hemi-SNPs that have been directly linkage mapped, both of which can be assigned to a genome, are shown as dark points. The broken blue and red horizontal lines mark significance $-\log 10 P=$ 5 and $-\log 10 P=4$, respectively

BnC07_0238 (Supplemental Figure 3g) respectively. The last three potential loci were found on A01: BnA01_0308 (Supplemental Figure 3a), A02: BnA02_0286 (Supplemental Figure 3c) and A08: BnA08_0009 (Supplemental Figure 3e).

Candidate genes from SNP analysis were searched in the total area of 392 genes (in LD blocks containing significant SNPs; Supplemental Figure 4 or at a distance of $0.2 \mathrm{Mb}$ from the pSNPs/sSNPs in locus). In these regions, potential candidate genes directly/ indirectly involved in clubroot resistance are identified Supplemental Table 2. Using the results of enrichment analysis, genes containing phrase "defence response", "ethylene", "jasmonic acid", "salicylic acid", "abscisic acid", "auxin" and "gibberellin" were considered as potential candidate genes. Based on the InterPro analysis and annotation, the transcription factors with plant defence domains ERF, bZIP, WRKY, MYB, plant defence cis-regulatory ET/JA motifs, G-box, GCC-box, W-box and pathogenrelated proteins were included to candidate gene group (Singh et al. 2002). The estimated narrowsense heritability $\left(h^{2}\right)$ of DI 0.469 suggests that genetic variability may play a substantial role in CR resistance. In total, 63 candidate genes have been identified from SNP analysis (Supplemental Table 2). 

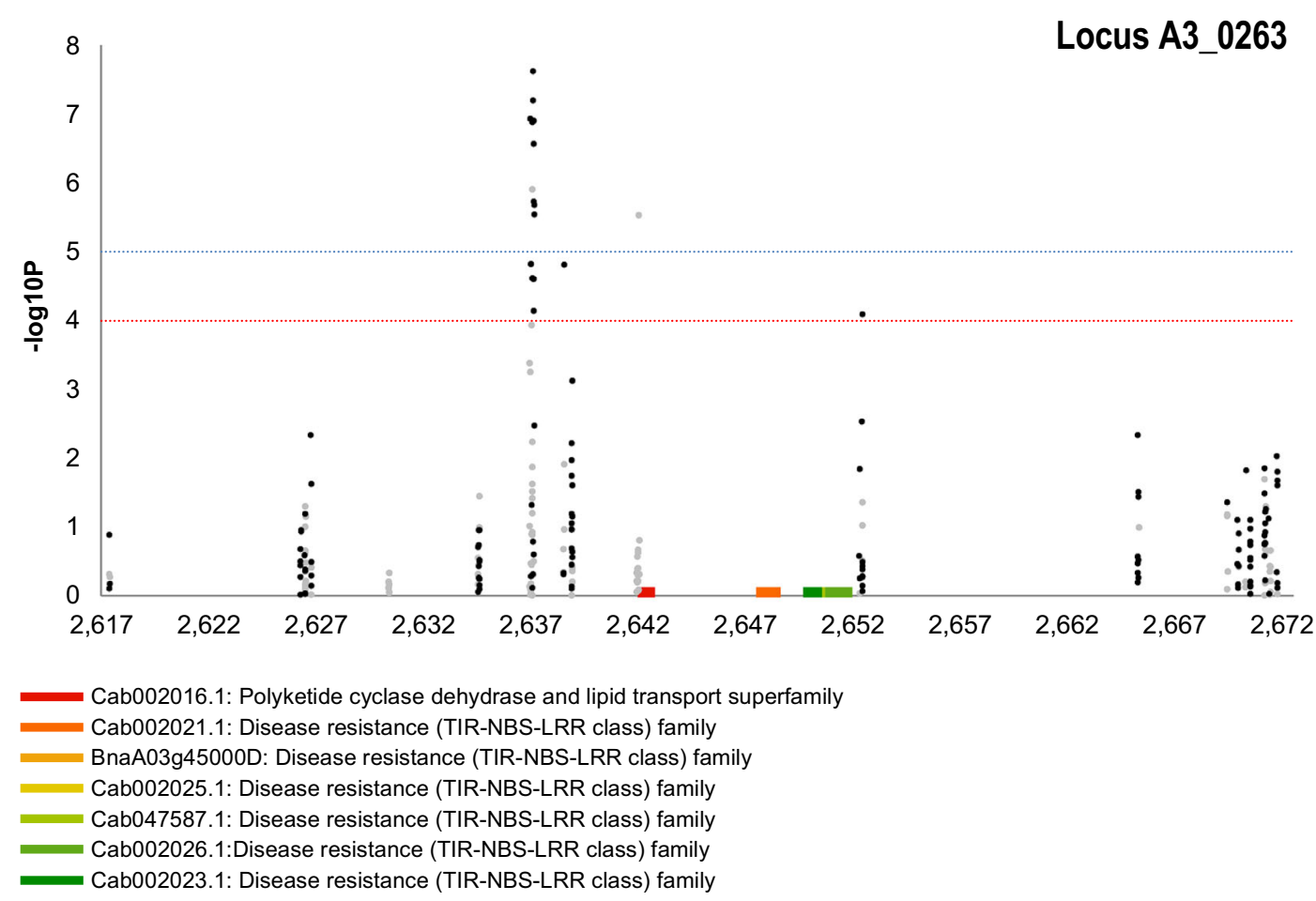

Fig. 4 SNP association analysis for clubroot resistance focus on part of pseudomolecule with the highest associated locus (around 26 million bases from the beginning of the A03 chromosome). The SNP are positioned on the $x$-axis based on their location (units $10^{5}$ ), the positions of the candidate genes for this locus are further indicated on $x$-axis. On the $y$-axis are values of the trait association significance $(-\log 10 P)$. The black signs represent simple SNP and hemi-SNP markers assigned to the corresponding genome and grey hemi-SNP markers for which the genome of the polymorphism cannot be assigned. The dashed blue and red lines mark significance $-\log 10 P=5$ and $-\log 10 P=4$, respectively

\section{GEMs analysis}

Candidate genes were identified on the significance limit of $-\log 10 P>3.5$. Graphic representation of the gene expression analysis is shown in Supplemental Figure 1. The gene expression analysis identified 21 genes which exceeded the defined limit of significance (Supplemental Table 3). In the total, we identified 21 genes above this limit (Supplemental Table 3). These genes were located on the chromosomes A05, A07, $\mathrm{A} 09, \mathrm{~A} 10, \mathrm{C} 02, \mathrm{C} 03, \mathrm{C} 04, \mathrm{C} 05, \mathrm{C} 06, \mathrm{C} 07, \mathrm{C} 08$ and C09 (Supplemental Figure 1). GO enrichment analysis, InterPro analysis and annotation were performed at the same way as for SNP candidate genes. Overall, 12 potential candidate genes have been identified from GEM analysis (Supplemental Table 3). These do not correspond to the positions of the SNP associations. This low number of candidates is a consequence of the available transcript abundance data being derived from leaf tissue, whereas the trait was measured in roots.

\section{Discussion}

Many studies have reported clubroot resistance loci in $B$. napus and its diploid progenitors $B$. rapa and B. oleracea, for example: Anju1, Anju2, Anju3, Anju4, $C R a, C r r A 5, C R b, C R b^{\text {kato }}, C R Q T L-G N \_1, C R Q T L-$ GN_2, CRc, CRd, Crr1, Crr2, Crr3_CRk, QTL_LG9, MCR-A4, MCR-C3, MCR-C9, PbBA31, PbBA32, SCRA10a, SCR-A10b, SCR-C3, SCR-C4a, SCR-C4b, SCRC6, Rcr1, Rcr2, Rcr4, Rcr7, Rcr8 and Rcr9 (Chen et al. 2013; Chu et al. 2014; Dakouri et al. 2018; Hayashida et al. 2008; Huang et al. 2017; Kato et al. 2013; Lee et al. 2016; Li et al. 2016; Nagaoka et al. 2010; Nguyen et al. 2018; Nomura et al. 2005; Pang et al. 2018; Saito et al. 2006; Sakamoto et al. 2008; Suwabe et al. 2003; Yu et al. 2017; Zhang et al. 2014). In this study, we aimed to use AT to identify further loci and candidate genes playing key roles in clubroot resistance in oilseed rape. The clubroot disease is difficult to control, once the soil is infested with spores of $P$. brassicae, the soil 
contamination could last for more than 20 years (Dixon 2009). In the last decade, this disease is spreading rapidly (Ricarova et al. 2017). In the light of seriousness, numerous studies have been conducted to discover resistance genes. However, most studies were carried out on B. rapa. In B. napus, a source of the major resistance gene has been found in Mendel variety, which shows resistance against certain pathogens. Unfortunately, the nature of resistance is based on only one resistance gene and it was overcome by new pathotype or high pathogen pressure (Diederichsen et al. 2014).

Recent GWAS analysis conducted in B. napus for Chinese pathotype 4 identified nine new resistance loci ( $\mathrm{Li}$ et al. 2016). In this study, we performed GWAS with a mix of European $P$. brassicae pathotypes $17 / 31 / 31$. We used a large diversity panel of $B$. napus representing genotypes from around the world. For association analysis, we combined SNP markers and transcript abundance from mRNA-Seq to detect new resistance loci and potential candidate genes for resistance against clubroot disease.

In total, we identified 86 SNPs to be highly associated with clubroot resistance. Twenty-nine of them with significance of $P<10^{-5}$ and 57 SNPs with significance $10^{-5}<P<10^{-4}$. SNPs were located into nine small loci (BnA01_0308,BnA02_0265, BnA02_0286, BnA03_0186, BnA03_0263, BnA08_0009, BnC02_0414, BnC07_0238, BnC07_0421). This suggests that the clubroot resistance is probably quantitatively inherited trait controlled by multiple loci.

The most prominent association peak was located on chromosome A03, locus BnA03_0263 (Fig. 4), with few hemi-SNPs (markers with ambiguous genome anchoring) present in homeologous position on chromosome C07, locus BnC07_0421 (Supplemental Figure 3h). Among the genes containing most of the sSNPs on chromosome A03, 7 candidate genes were found, of which 6 belong to the Disease Resistance (TIR-NBSLRR class) family. In the corresponding region of $B$. rapa genome or in close proximity, resistance genes $C R a, C R b^{\text {kato }}, R c r 1, R c r 2$ and $R c r 4$ have been identified (Hayashida et al. 2008; Ueno et al. 2012; Kato et al. 2013; Chu et al. 2014; Huang et al. 2017; Yu et al. 2017). The GEM analysis revealed among 6 disease resistance candidates, one with a high correlation with the clubroot DI (BnaA03g45000D). This gene shows the greatest similarity with the already cloned $C R a$ clubroot resistance gene in the $B$. rapa genome. However, in the study by Zhang et al. (2016) focusing specifically on $C R a$ ortholog in B. napus, localized in high proximity of our locus BnA03_0263, the same principle of resistance has not been demonstrated and it has been suggested that resistance to $P$. brassicae may be controlled by the combined effect of a new CR gene and $C R a$ from B. rapa (Zhang et al. 2016).

Another associated locus BnA03_0186 (Supplemental Figure 3d) has been detected on chromosome A03. We found 7 candidate genes directly containing SSNPs lying in close proximity of a group of 4 leucine-rich repeat transmembrane kinase genes. The ortholog of this gene in A. thaliana is localized to the plasma membrane, and it is involved in the regulation of plant innate immunity. Moreover, this gene has the ability to recognize chitin (Le et al. 2014) and its transcription is strongly declined under clubroot infection (Siemens et al. 2006). Another candidate gene containing pSNPs is ERF domain 11; ethylene response factor acts as a negative regulator of JA-responsive defence gene expression, resistance to fungal pathogen Fusarium oxysporum and antagonist of JA inhibition of root elongation (Lyons et al. 2013).

The second most prominent association peak was located on chromosome A02, locus BnA02_0265 (Fig. 5). In this locus, we identified 9 candidate genes. The most promising are Tryptophan RNA-binding attenuator contains SSNPs. A. thaliana ortholog is in the direct interaction with PEN3 required for non-host resistance (Campe et al. 2016) and pectin lyase-like superfamily also contains sSNPs. Its role is cell wall modification (Etchells et al. 2012). This locus also included RING U-box superfamily, which is disease resistance protein (TIR-NBS class) with function of signal transduction, apoptosis and innate immune response. Other candidate genes can be seen below.

A second locus on A02 chromosome BnA02_0286 contains two candidate genes, A. thaliana orthologs cytochrome P450, family 71, subfamily B, polypeptide 20 (potential genetic target of Whirly transcription factor) and defence response CAP-gly domain linker.

Loci BnA02_0265 and BnA02_0286 overlap a relatively large locus designed as $\operatorname{Rcr} 8$ (Yu et al. 2017).

Another associated locus with pSNPs was found on chromosome A01. Within this locus, 11 candidate genes are present and their position is not overlapping with previously described region where $C r r 2$ gene has been identified in B. rapa (Suwabe et al. 2003). The direct gene encompassing pSNP is an ortholog ATPase, AAAtype, CDC48 and negative regulator of NLR-mediated 


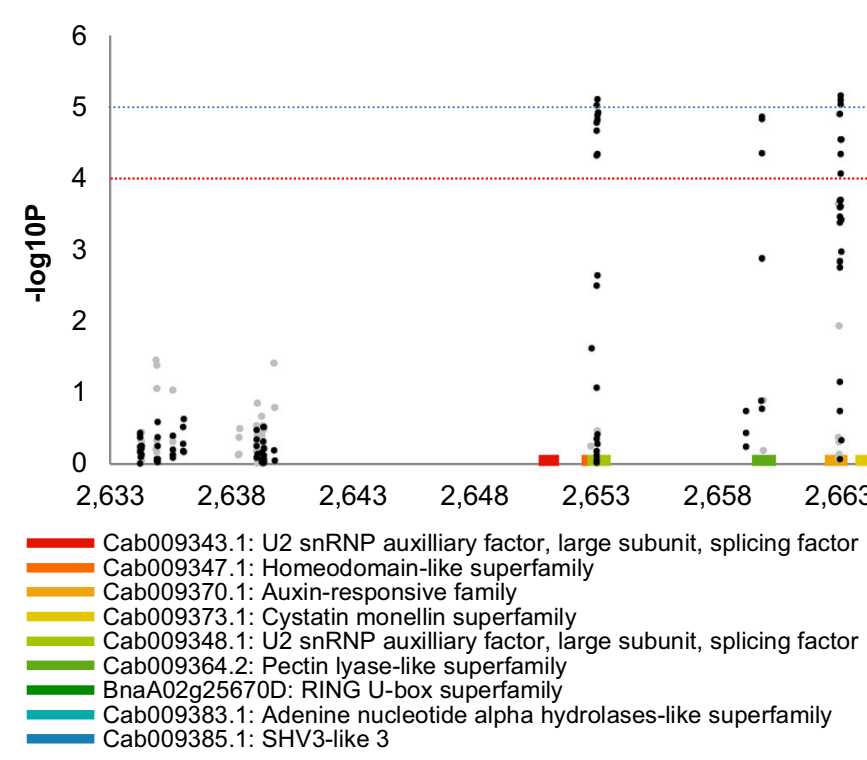

Fig. 5 SNP association analysis for clubroot resistance focus on part of pseudomolecule with the second most prominent association peak (around 26 million bases from the beginning of the A02 chromosome). The SNP are positioned on the $x$-axis based on their location (units $10^{5}$ ), the positions of the candidate genes for this locus are further indicated on $x$-axis. On the $y$-axis are values of the

immunity (Copeland et al. 2016). Next to this gene is another interesting candidate gene ortholog of PATTERN-TRIGGERED IMMUNITY (PTI) COMPROMISED RECEPTOR-LIKE CYTOPLASMIC KINASE 1 and $P C R K 1$. This gene is important for immunity induced by damage associated molecular patternDAMPs (Sreekanta et al. 2015a, b). Interesting gene in this locus is also phytosulphokine 5 precursor, which is an important signalling in resistance in root infection of Fusarium oxysporum (Shen and Diener 2013).

The other associated locus was found on chromosome A8. This does not correspond to resistance loci identified previously on this chromosome, $\operatorname{Rcr} 9$ and $\mathrm{Crr}$ l, being in different positions on the chromosome (Yu et al. 2017; Suwabe et al. 2003). It contains 6 candidate genes, for example cluster of orthologs of GDSL-motif lipase 2 and GDSL-like Lipase Acylhydrolase superfamily. They are involved in disease resistance and negatively regulate auxin signalling (Lee et al. 2009). GDSL-like lipase is also engaged in defence against Alternaria brassicicola (Oh et al. 2005).

GEM association revealed 21 genes, exceeding the limit of $-\log 10 \mathrm{p} 3.5$. These genes were distributed across the whole genome, with slight predominance towards $\mathrm{C}$ genome. Performing GO enrichment trait association significance $(-\log 10 P)$. The black signs represent simple SNP and hemi-SNP markers assigned to the corresponding genome and grey hemi-SNP markers for which the genome of the polymorphism cannot be assigned. The dashed blue and red lines mark significance $-\log 10 P=5$ and $-\log 10 P=4$, respectively

analysis, InterPro analysis and using blastn for annotation gene with orthologs of $A$. thaliana, we reduced the number of candidate genes to nineteen. The most promising candidates were orthologs of pentacyclic triterpene synthase 1, which is important for of PEN1 and PEN2 functions in powdery mildew non-host interaction. This synthase mediates transport required for innate immunity and focal accumulation of syntaxin PEN1 (Nielsen et al. 2012). Another candidate cytokinin response factor 2 plays important role in response to stress condition and auxin regulation (Simackova et al. 2015). S-adenosyl-L-methioninedependent methyltransferase superfamily is another stress candidate gene especially important in drought tolerance (Nir et al. 2014). It is important to recognize that only genes with expression in the source tissue for mRNAseq (i.e. leaves) correlated with the CR trait can be identified as GEM associations; those with root-specific or infection-specific expression cannot be identified by AT with the expression data available. However, AT analysis revealed candidate genes directly or indirectly involved in clubroot resistance, not only as results of the significant association between DI and sequence variation present in our diversity panel, but also as result of difference in 
transcript abundance within the panel and therefore provides added value in the association analysis.

Translation of our findings into improved clubroot resistance of new B. napus varieties will involve the development of molecular markers to select alleles associated with greater resistance. To aid this, we have compiled (Supplemental Figure 5) shortlists of suitable polymorphisms to underpin the development of high throughput SNP markers. The use of molecular markers to pre-select seedling for trialling will improve the speed and efficiency of breeding for clubroot resistance.

Funding information This work was supported by The Czech National Agency for Agricultural Research (NAZV QJ1510172 and QK1910070) and UK Biotechnology and Biological Sciences Research Council (BB/L002124/1).

Open Access This article is distributed under the terms of the Creative Commons Attribution 4.0 International License (http:// creativecommons.org/licenses/by/4.0/), which permits unrestricted use, distribution, and reproduction in any medium, provided you give appropriate credit to the original author(s) and the source, provide a link to the Creative Commons license, and indicate if changes were made.

\section{References}

Alcock TD, Havlickova L, He Z, Bancroft I, White PJ, Broadley MR, Graham N (2017) Identification of candidate genes for calcium and magnesium accumulation in Brassica napus L. by association genetics. Front. Plant Sci 8:1968. https://doi. org/10.3389/fpls.2017.01968

Bhattacharya I, Dutta S, Mondal S, Mondal B (2014) Clubroot disease on Brassica crops in India. Can J Plant Pathol 36: 154-160. https://doi.org/10.1080/07060661.2013.875064

Buczacki ST, Toxopeus H, Mattusch P, Johnston TD, Dixon GR, Hobolth LA (1975) Study of physiologic specialization in Plasmodiophora Brassicae: proposals for attempted rationalization through an international approach. Trans Br Mycol Soc 65:295-303. https://doi.org/10.1016/S0007-1536(75 )80013-1

Campe R, Langenbach C, Leissing F, Popescu GV, Popescu SC, Goellner K, Beckers GJM, Conrath U (2016) ABC transporter PEN3/PDR8/ABCG36 interacts with calmodulin that, like PEN3, is required for Arabidopsis nonhost resistance. New Phytol 209:294-306. https://doi.org/10.1111/nph.13582

Chai AL, Xie XW, Shi YX, Li BJ (2014) Research status of clubroot (Plasmodiophora Brassicae) on cruciferous crops in China. Can J Plant Pathol 36:142-153. https://doi. org/10.1080/07060661.2013.868829

Chen J, Jing J, Zhan Z, Zhang T, Zhang C, Piao Z (2013) Identification of novel QTLs for isolate-specific partial resistance to Plasmodiophora brassicae in Brassica rapa. PLoS
One 8:e85307. https://doi.org/10.1371/journal. pone. 0085307

Chu M, Song T, Falk KC, Zhang X, Liu X, Chang A, Lahlali R, McGregor L, Gossen BD, Yu F, Peng G (2014) Fine mapping of Rcrl and analyses of its effect on transcriptome patterns during infection by Plasmodiophora brassicae. BMC Genomics 15:1166. https://doi.org/10.1186/1471-2164-151166

Copeland C, Woloshen V, Huang Y, Li X (2016) AtCDC48A is involved in the turnover of an NLR immune receptor. Plant $\mathrm{J}$ 88:294-305. https://doi.org/10.1111/tpj.13251

Dakouri A, Zhang X, Peng G, Falk KC, Gossen BD, Strelkov SE, Yu F (2018) Analysis of genome-wide variants through bulked segregant RNA sequencing reveals a major gene for resistance to Plasmodiophora Brassicae in Brassica oleracea. Sci Rep 8:17657. https://doi.org/10.1038/s41598018-36187-5

Diederichsen E, Frauen M, Ludwig-Mueller J (2014) Clubroot disease management challenges from a German perspective. Can J Plant Pathol 36:85-98. https://doi.org/10.1080 /07060661.2013.861871

Dixon GR (2009) Plasmodiophora Brassicae in its environment. J Plant Growth Regul 28:212-228. https://doi.org/10.1007 /s00344-009-9098-3

Donald EC, Porter IJ (2014) Clubroot in Australia: the history and impact of Plasmodiophora brassicae in Brassica crops and research efforts directed towards its control. Can J Plant Pathol 36:66-84. https://doi.org/10.1080 /07060661.2013.873482

Etchells JP, Moore L, Jiang WZ, Prescott H, Capper R, Saunders NJ, Bhatt AM, Dickinson HG (2012) A role for BELLRINGER in cell wall development is supported by loss-of-function phenotypes. BMC Plant Biol 12:212. https://doi.org/10.1186/1471-2229-12-212

Faggian R, Strelkov SE (2009) Detection and Measurement of Plasmodiophora brassicae. J Plant Growth Regul 28(3):282288

Fredua-Agyeman R, Rahman H (2016) Mapping of the clubroot disease resistance in spring Brassica napus canola introgressed from European winter canola Cv. 'Mendel.'. Euphytica 211:201-213. https://doi.org/10.1007/s10681016-1730-2

Grandclément C, Thomas G (1996) Detection and analysis of QTLs based on RAPD markers for polygenic resistance to Plasmodiophora brassicae Woron in Brassica oleracea L. Theor Appl Genet 93:86-90. https://doi.org/10.1007 /BF00225731

Harper AL, Trick M, Higgins J, Fraser F, Clissold L, Wells R, Hattori C, Werner P, Bancroft I (2012) Associative transcriptomics of traits in the polyploid crop species Brassica napus. Nat Biotechnol 30:798-802. https://doi.org/10.1038 /nbt. 2302

Hatakeyama K, Suwabe K, Tomita RN, Kato T, Nunome T, Fukuoka H, Matsumoto S (2013) Identification and characterization of Crrla, a gene for resistance to clubroot disease (Plasmodiophora brassicae Woronin) in Brassica rapa L. PLoS One 8:e54745. https://doi.org/10.1371/journal. pone. 0054745

Havlickova L, He Z, Wang L, Langer S, Harper AL, Kaur H, Broadley MR, Gegas V, Bancroft I (2018) Validation of an updated associative transcriptomics platform for the 
polyploid crop species Brassica napus by dissection of the genetic architecture of erucic acid and tocopherol isoform variation in seeds. Plant J 93:181-192. https://doi. org/10.1111/tpj.13767

Hayashida N, Takabatake Y, Nakazawa N, Aruga D, Nakanishi H, Taguchi G, Sakamoto K, Matsumoto E (2008) Construction of a practical SCAR marker linked to clubroot resistance in Chinese cabbage, with intensive analysis of HC352b genes. J Jpn Soc Hortic Sci 77:150-154. https://doi.org/10.2503 /jjshs 1.77 .150

He Z, Wang L, Harper AL, Havlickova L, Pradhan AK, Parkin IAP, Bancroft I (2017) Extensive homoeologous genome exchanges in allopolyploid crops revealed by mRNAseqbased visualization. Plant Biotechnol J 15(5):594-604

Hirai M (2006) Genetic analysis of clubroot resistance in Brassica crops. Breed Sci 56:223-229. https://doi.org/10.1270 /jsbbs.56.223

Huang Z, Peng G, Liu X, Deora A, Falk KC, Gossen BD, McDonald MR, Yu F (2017) Fine mapping of a clubroot resistance gene in chinese cabbage using snp markers identified from bulked segregant RNA sequencing. Front Plant Sci 8. https://doi.org/10.3389/fpls.2017.01448

Hwang SF, Strelkov SE, Feng J, Gossen BD, Howard RJ (2012) Plasmodiophora brassicae: a review of an emerging pathogen of the Canadian canola (Brassica napus) crop. Mol Plant Pathol 13:105-113. https://doi.org/10.1111/j.13643703.2011.00729.x

Hwang SF, Ahmed HU, Zhou Q, Strelkov SE, Gossen BD, Peng G, Turnbull GD (2014) Efficacy of Vapam fumigant against clubroot (Plasmodiophora brassicae) of canola. Plant Pathol 63:1374-1383. https://doi.org/10.1111/ppa.12207

Kageyama K, Asano T (2009) Life cycle of Plasmodiophora Brassicae. J Plant Growth Regul 28:203-211. https://doi. org/10.1007/s00344-009-9101-z

Kato T, Hatakeyama K, Fukino N, Matsumoto S (2013) Fine mapping of the clubroot resistance gene $C R b$ and development of a useful selectable marker in Brassica rapa. Breed Sci 63:116-124. https://doi.org/10.1270/jsbbs.63.116

Koprivova A, Harper AL, Trick M, Bancroft I, Kopriva S (2014) Dissection of the control of anion homeostasis by associative transcriptomics in Brassica napus. Plant Physiol 166:442450. https://doi.org/10.1104/pp.114.239947

Landry BS, Hubert N, Crete R, Chang MS, Lincoln SE, Etoh T (1992) A genetic map for Brassica oleracea based on RFLP markers detected with expressed DNA sequences and mapping of resistance genes to race 2 of Plasmodiophora brassicae (Woronin). Genome 35:409-420. https://doi. org/10.1139/g92-061

Le MH, Cao Y, Zhang XC, Stacey C (2014) LIK1, A CERK1interacting kinase, regulates plant immune responses in Arabidopsis. PLoS One 9:e102245. https://doi.org/10.1371 /journal.pone.0102245

Lee DS, Kim BK, Kwon SJ, Jin HC, Park OK (2009) Arabidopsis GDSL lipase 2 plays a role in pathogen defense via negative regulation of auxin signaling. Biochem Biophys Res Commun 379:1038-1042. https://doi.org/10.1016/j. bbrc.2009.01.006

Lee J, Izzah NK, Choi BS, Joh HJ, Lee SC, Perumal S, Seo J, Ahn K, Jo EJ, Choi GJ, Nou IS, Yu Y, Yang TJ (2016) Genotyping-by-sequencing map permits identification of clubroot resistance QTLs and revision of the reference genome assembly in cabbage (Brassica oleracea L.). DNA Res 23:29-41. https://doi.org/10.1093/dnares/dsv034

Li L, Luo Y, Chen B, Xu K, Zhang F, Li H, Huang Q, Xiao X, Zhang T, Hu J, Li F, Wu X (2016) A genome-wide association study reveals new loci for resistance to clubroot disease in Brassica napus. Front Plant Sci 7:1483. https://doi. org/10.3389/fpls.2016.01483

Lu G, Harper AL, Trick M, Morgan C, Fraser F, O’Neill C, Bancroft I (2014) Associative transcriptomics study dissects the genetic architecture of seed glucosinolate content in Brassica napus. DNA Res 21:613-625. https://doi. org/10.1093/dnares/dsu024

Lyons R, Iwase A, Gänsewig T, Sherstnev A, Duc C, Barton GJ, Hanada K, Higuchi-Takeuchi M, Matsui M, Sugimoto K, Kazan K, Simpson GG, Shirasu K (2013) The RNAbinding protein FPA regulates Flg22-triggered defense responses and transcription factor activity by alternative polyadenylation. Sci Rep 3:2866. https://doi.org/10.1038 /srep02866

Manzanares-Dauleux MJ, Divaret I, Baron F, Thomas G (2000) Evaluation of French Brassica oleracea landraces for resistance to Plasmodiophora brassicae. Euphytica 113:211-218. https://oi.org/10.1023/A:1003997421340

Nagaoka T, Doullah MAU, Matsumoto S, Kawasaki S, Ishikawa T, Hori H, Okazaki K (2010) Identification of QTLs that control clubroot resistance in Brassica oleracea and comparative analysis of clubroot resistance genes between $B$. rapa and B. oleracea. Theor Appl Genet 120:1335-1346. https://doi.org/10.1007/s00122-010-1259-z

Nguyen ML, Monakhos GF, Komakhin RA, Monakhos SG (2018) The new Clubroot resistance locus is located on chromosome A05 in Chinese cabbage (Brassica rapa L.). Russ J Genet 54:296-304. https://doi.org/10.1134 /S1022795418030080

Nielsen ME, Feechan A, Böhlenius H, Ueda T, ThordalChristensen H (2012) Arabidopsis ARF-GTP exchange factor, GNOM, mediates transport required for innate immunity and focal accumulation of syntaxin PEN1. Proc Natl Acad Sci U S A 109:11443-11448. https://doi.org/10.1073 /pnas.1117596109

Nir I, Moshelion M, Weiss D (2014) The Arabidopsis GIBBERELLIN METHYL TRANSFERASE 1 suppresses gibberellin activity, reduces whole-plant transpiration and promotes drought tolerance in transgenic tomato. Plant Cell Environ 37:113-123. https://doi.org/10.1111/pce.12135

Nomura K, Minegishi Y, Kimizuka-Takagi C, Fujioka T, Moriguchi K, Shishido R, Ikehashi H (2005) Evaluation of F2 and F3 plants introgressed with QTLs for clubroot resistance in cabbage developed by using SCAR markers. Plant Breed 124:371-375. https://doi.org/10.1111/j.14390523.2005.01105.x

Oh IS, Park AR, Bae MS, Kwon SJ, Kim YS, Lee JE, Kang NY, Lee S, Cheong H, Park OK (2005) Secretome analysis reveals an Arabidopsis lipase involved in defense against Alternaria brassicicola. Plant Cell 17:2832-2847. https://doi.org/10.1105/tpc.105.034819

Pageau K, Reisdorf-Cren M, Morot-Gaudry JF, MasclauxDaubresse C (2006) The two senescence-related markers, GS1 (cytosolic glutamine synthetase) and GDH (glutamate dehydrogenase), involved in nitrogen mobilization, are differentially regulated during pathogen attack and by stress 
hormones and reactive oxygen species in Nicotiana tabacum L. leaves. J Exp Bot 57:547-557. https://doi.org/10.1093 /jxb/erj035

Pang W, Fu P, Li X, Zhan Z, Yu S, Piao Z (2018) Identification and mapping of the clubroot resistance gene CRd in Chinese cabbage (Brassica Rapa ssp. Pekinensis). Front Plant Sci 9. https://doi.org/10.3389/fpls.2018.00653

Piao Z, Ramchiary N, Lim YP (2009) Genetics of clubroot resistance in Brassica species. J Plant Growth Regul 28:252-264. https://doi.org/10.1007/s00344-009-9093-8

Rahman H, Peng G, Yu F, Falk KC, Kulkarni M, Selvaraj G (2014) Genetics and breeding for clubroot resistance in canadian spring canola (Brassica napus L.). Can J Plant Pathol 36: 122-134. https://doi.org/10.1080/07060661.2013.862571

Ricarova V, Kazda J, Baranyk P, Rysanek P (2016) Clubroot - an emerging disease faced by Czech oilseed rape growers. Sci Agric Bohem 47:105-112. https://doi.org/10.1515/sab-20160015

Ricarova V, Kazda J, Baranyk P, Rysanek P (2017) Greenhouse and field experiments with winter oilseed rape cultivars resistant to Plasmodiophora brassicae Wor. Crop Prot 92:6069. https://doi.org/10.1016/j.cropro.2016.10.009

Rocherieux J, Glory P, Giboulot A, Boury S, Barbeyron G, Thomas G, Manzanares-Dauleux MJ (2004) Isolate-specific and broad-spectrum QTLs are involved in the control of clubroot in Brassica oleracea. Theor Appl Genet 108: 1555-1563. https://doi.org/10.1007/s00122-003-1580-x

Saito M, Kubo N, Matsumoto S, Suwabe K, Tsukada M, Hirai M (2006) Fine mapping of the clubroot resistance gene, Crr3, in Brassica rapa. Theor Appl Genet 114:81-91. https://doi. org/10.1007/s00122-006-0412-1

Sakamoto K, Saito A, Hayashida N, Taguchi G, Matsumoto E (2008) Mapping of isolate-specific QTLs for clubroot resistance in Chinese cabbage (Brassica rapa L. ssp. pekinensis). Theor Appl Genet 117:759-767. https://doi.org/10.1007 /s00122-008-0817-0

Samayoa LF, Malvar RA, Olukolu BA, Holland JB, Butron A (2015) Genome-wide association study reveals a set of genes associated with resistance to the Mediterranean corn borer (Sesamia nonagrioides $\mathrm{L}$ ) in a maize diversity panel. BMC Plant Biol 15:350. https://doi.org/10.1186/s12870-014-04033

Shen Y, Diener AC (2013) Arabidopsis thaliana RESISTANCE TO FUSARIUM OXYSPORUM 2 implicates tyrosinesulfated peptide signaling in susceptibility and resistance to root infection. PLoS Genet 9:e1003525. https://doi. org/10.1371/journal.pgen.1003525

Siemens J, Keller I, Sarx J, Kunz S, Schuller A, Nagel W, Schmülling T, Parniske M, Ludwig-Müller J (2006) Transcriptome analysis of Arabidopsis clubroots indicate a key role for cytokinins in disease development. Mol PlantMicrobe Interact 19:480-494. https://doi.org/10.1094 /MPMI-19-0480

Simackova M, O'Brien JA, Khan M, Noorden GV, Otvos K, Vieten A, De Clercq I, Van Haperen JMA, Cuesta C, Hoyerová K, Vanneste S, Mrhavy P, Wabnik K, Van Breusegem F, Nowack M, Murphy A, Friml J, Weijeres D, Beeckman T, Benková E (2015) Cytokinin response factors regulate PIN-FORMED auxin transporters. Nat Commun 6: 8717. https://doi.org/10.1038/ncomms9717
Singh K, Foley RC, Onate-Sanchez L (2002) Transcription factors in plant defense and stress responses. Curr Opin Plant Biol 5: 430-436. https://doi.org/10.1016/S1369-5266(02)00289-3

Some A, Manzanares MJ, Laurens F, Baron F, Thomas G, Rouxel F (1996) Variation for virulence on Brassica napus L. amongst Plasmodiophora brassicae collections from France and derived single-spore isolates. Plant Pathol 45: 432-439. https://doi.org/10.1046/j.1365-3059.1996.d01155.x

Sreekanta S, Bethke G, Hatsugai N, Tsuda K, Thao A, Wang L, Katagiri F, Glazebrook J (2015a) The receptor-like cytoplasmic kinase $P C R K 1$ contributes to pattern-triggered immunity against Pseudomonas syringae in Arabidopsis thaliana. New Phytol 207:78-90. https://doi.org/10.1111/nph.13345

Sreekanta S, Haruta M, Minkoff BB, Glazebrook J (2015b) Functional characterization of $P C R K 1$, a putative protein kinase with a role in immunity. Plant Signal Behav 10: e 1063759 -e 1063751 . https://doi.org/10.1080 $/ 15592324.2015 .1063759$

Suwabe K, Tsukazaki H, Iketani H, Hatakeyama K, Fujimura M, Nunome T, Fukuoka H, Matsumoto S, Hirai M (2003) Identification of two loci for resistance to clubroot (Plasmodiophora brassicae Woronin) in Brassica rapa L. Theor Appl Genet 107:997-1002. https://doi.org/10.1007 /s00122-003-1309-x

Suwabe K, Tsukazaki H, Iketani H, Hatakeyama K, Kondo M, Fujimura M, Nunome T, Fukuoka H, Hirai M, Matsumoto S (2006) Simple sequence repeat-based comparative genomics between Brassica rapa and Arabidopsis thaliana: the genetic origin of clubroot resistance. Genetics 173:309-319. https://doi.org/10.1534/genetics.104.038968

Ueno H, Matsumoto E, Aruga D, Kitagawa S, Matsumura H, Hayashida N (2012) Molecular characterization of the $C R a$ gene conferring clubroot resistance in Brassica rapa. Plant Mol Biol 80:621-629. https://doi.org/10.1007/s11103-0129971-5

Voorrips RE, Jongerius MC, Kanne HJ (1997) Mapping of two genes for resistance to clubroot (Plasmodiophora brassicae) in a population of doubled haploid lines of Brassica oleracea by means of RFLP and AFLP markers. Theor Appl Genet 94: 75-82. https://doi.org/10.1007/s001220050384

Wallenhammar AC (1996) Prevalence of Plasmodiophora brassicae in a spring oilseed rape growing area in central Sweden and factors influencing soil infestation levels. Plant Pathol 45:710-719. https://doi.org/10.1046/j.13653059.1996.d01-173.x

Werner S, Diederichsen E, Frauen M, Schondelmaier J, Jung C (2008) Genetic mapping of clubroot resistance genes in oilseed rape. Theor Appl Genet 116:363-372. https://doi. org/10.1007/s00122-007-0674-2

Williams PH (1966) A system for the determination of races of Plasmodiophora brassicae that infect cabbage and rutabaga. Phytopathology 56:624-626

Wood IP, Pearson BM, Garcia-Gutierrez EG, Havlickova L, He Z, Harper AL, Bancroft I, Waldron KW (2017) Carbohydrate microarrays and their use for the identification of molecular markers for plant cell wall composition. Proc Natl Acad Sci U S A 114:6860-6865. https://doi.org/10.1073 /pnas.1619033114

Yu F, Zhang X, Peng G, Falk KC, Strelkov SE, Gossen BD (2017) Genotyping-by-sequencing reveals three QTL for clubroot 
resistance to six pathotypes of Plasmodiophora Brassicae in Brassica rapa. Sci Rep 7:4516. https://doi.org/10.1038 /s41598-017-04903-2

Zhang T, Zhao Z, Zhang C, Pang W, Choi SR, Lim YP, Piao Z (2014) Fine genetic and physical mapping of the $C R b$ gene conferring resistance to clubroot disease in Brassica rapa. Mol Breed 34:1173-1183. https://doi.org/10.1007/s11032014-0108-1
Zhang H, Feng J, Hwang SF, Strelkov SE, Falak I, Huang X, Sun R (2016) Mapping of clubroot (Plasmodiophora brassicae) resistance in canola (Brassica napus). Plant Pathol 65:435440. https://doi.org/10.1111/ppa.12422

Publisher's note Springer Nature remains neutral with regard to jurisdictional claims in published maps and institutional affiliations. 